\title{
NON-DESTRUCTIVE INSPECTION AND MONITORING OF FRACTURES IN CONCRETE WITH SELF-HEALING PROPERTIES
}

\author{
Fabian Malm $^{1}$, Fabian Diewald $^{2}, \underline{\text { Katja Pinkert }}^{1}$ \\ ${ }^{1}$ Chair of Nondestructive Testing, Centre for Building Materials, Technical \\ University of Munich, Baumbachstr. 7, 81245, Munich, Germany, Email: \\ katja.pinkert@tum.de \\ ${ }^{2}$ Chair of Materials Science and Testing, Centre for Building Materials, Technical \\ University of Munich, Baumbachstr. 7, 81245, Munich, Germany, Email: \\ fabian.diewald@tum.de
}

\begin{abstract}
Studying the properties of self-healing concrete, non-destructive testing techniques are beneficial to monitor the opening of cracks under load as well as the activation and efficiency of healing agents. The localization of defects and activation of self-healing agents, e.g. capturing the breakage of the encapsulation, is realized by the acoustic emission analysis (AEA) during the mechanical loading of concrete beams. The rebind of crack flanks due to an adhesive self-healing agent is verified by AEA and ultrasonic time of flight (ToF) method. Several proof-of-concept experiments were performed for test samples with selectively initiated fractures. To enhance the durability of infrastructure constructions by predictive maintenance of heterogeneous, self-healing components, a further step in signal analysis must be taken to evaluate online monitoring results even for unpredicted initial micro-crack formation and selective healing. Ultrasound signal correlation analysis is discussed regarding damage detection as well as the verification of self-healing properties.
\end{abstract}

Keywords: Self-healing concrete, non-destructive test, Acoustic emission analysis, ultrasonic time of flight

\section{INTRODUCTION}

Sustainability of infrastructure constructions - buildings, streets, railways, tunnels and bridges - is indispensable for our life as our mobility, industrial performance and functionality of modern society in general depend on the reliability and availability of these structures and their construction materials. About $70 \%$ of infrastructure constructions are built of concrete. Reinforced concrete, a cracked material, however, suffers from concrete cracks larger than $0.2 \mathrm{~mm}$ to $0.4 \mathrm{~mm}$, caused by shrinkage, freezing and thawing, thermal actions, differential settlements or mechanical loading. These cracks restrict the durability of the affected construction element (Fig. 1) if water impermeability can no longer be guaranteed. As a consequence, material defects result, e.g. caused by water induced cementitious hydration reactions, temperature and shrinkage effects, as well as aggressive corrosion of the reinforcement induced by the penetration of chlorides and CO2. (Snoeck et al., 2018) 


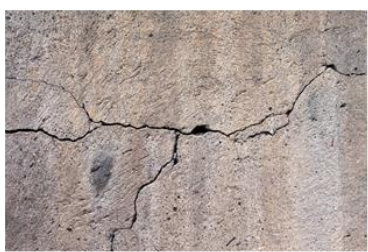

Crack formation and degradation

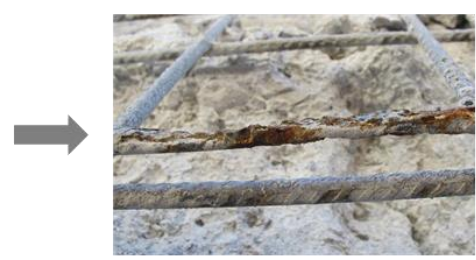

Corrosion of the reinforcement

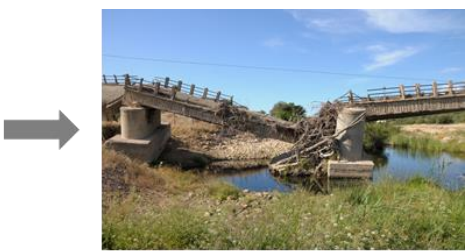

Structural failure

Figure 1. Degradation process of concrete supporting structures.

Natural and spontaneous fluid flow migration within cracks occurring in construction elements, on the other hand, enables autogenous self-healing of cementitious materials to a certain extent. Water penetration in open cracks also causes the dissolution of building materials calcium compounds. Transported by capillary forces into the interior of the crack system, these compounds precipitate and form protective layers or even close initial crack formations. An intervention of this process to promote and regulate the self-healing ability of construction materials could be performed by the addition of encapsulated healing agents. These agents should improve the autogenous and autonomous crack closure and create durable and sustainable concrete structures. (Snoeck et al., 2018)

Non-destructive testing techniques, in detail acoustic emission analysis and ultrasonic ToF method (Planès, Larose, 2013a), are established to monitor cementitious test specimensduring fracture formation and healing agent release, as well as before and after the damage and self-healing process. The investigation of heterogeneous materials by the determination of changes in travel time is, however, limited for detectable microscopic defect sizes and the number of occurring microscale defects. For concrete specimen, the size of heterogeneities is in the same order of magnitude as the wavelength of the traveling waves. Therefore, multiple scattering effects occur resulting in a diffuse wave field rather than a direct wave path. Beside the described investigation of the travel time by picking the onset of the detected wave mode, a comparison of the coda of the propagated elastic wave is stated. Linear correlation of the signal recorded during a mechanical load-induced damage as well as the healing process, to the signal recorded for the initial state is a powerful analysis tool to reveal even minimal structural changes, like initial microcracks. (Snoeck et al., 2018)

\section{SELF-HEALING CONCRETE}

Self-healing construction materials are categorized by the type of healing additive into "autogenous" healing and "autonomous" healing. These additives must become active as soon as the structure cracks. The concept of "autonomous" self-healing comprises the addition of material components that are not part of the original cement composition. Beside microencapsulated $\mathrm{CaCO} 3$-precipitating bacteria, adhesive components are investigated. (Snoeck et al., 2018, Hilloulin et al., 2015, De Rooij et al., 2013)

\section{Adhesive Self-Healing Agents}

Polymer-based adhesives are applied for self-healing of concrete structures to seal cracks and enable the force-locking connection of crack flanks (Fig 2).

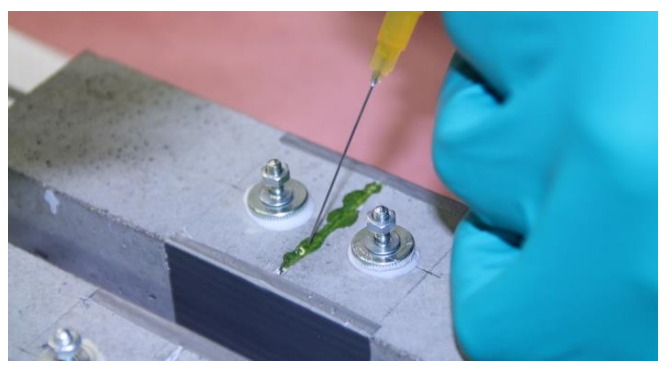


Figure 2. Manual application of a polymer-based adhesive for "autonomous" repair. (Crack width $\mathrm{d} \sim 0.3 \mathrm{~mm}$ ).

These adhesives are either one or more component systems. Adhesive characteristics like hardening, dispersal or wetting behaviour should be adapted to the specific use case. Polyurethanes or epoxy resins for example are adapted to dynamically loaded cracks due to their elastically flexible and ductile characteristics.

To introduce adhesive healing components within concrete structures, encapsulation technology is required. The release of the self-healing agent must be triggered by an emerging crack. A proof-of-concept-test shows the applicability of glass tubes to encapsulate adhesive components (Fig.3). Further developments for a broad application of this self-healing concept must yield at novel microcapsule techniques. (Feiteira, Gruyaert, De Belie, 2015, Zhang et al, 2013)

\section{NON-DESTRUCTIVE INSPECTION AND MONITORING}

The detailed study of damage and self-healing processes in building materials aims at the development of new construction materials with reliable self-healing properties as well as the predictive maintenance of sustainable infrastructure structures. The evaluation of crack formation and propagation in concrete, the monitoring of the release of healing agent and the investigation of loss or regain of properties, e.g. water tightness and force-locking connection of crack flanks, during the healing process at laboratory scales reveal insights into the performance of self-healing agents. Despite these initial investigations, a proof of activation of the healing mechanism in large components and the determination of the in-situ healing efficiency must be verified (Snoeck et al., 2018). An adequate testing method allows parallel loading and testing, the correlation of discrete load states of the specimen and its damage state, as well as the impact of an applied healing agent to a prolonged durability.

\section{Acoustic Emission Analysis}

Mechanical waves originating from crack formation and crack propagation in concrete structures are recorded and analysed for the monitoring of mechanical or thermal load scenarios. The passive testing method is always linked to an irreversible structural damage. However, analysing the recorded signals reveals characteristics of the emitting wave source, like location of the initial crack formation and fracture behaviour. A typical sensor setup for the acoustic emission analysis during the mechanical load scenario of a three point-bending test is shown in Figure 3. (Flohr, Malm, Grosse, 2017)
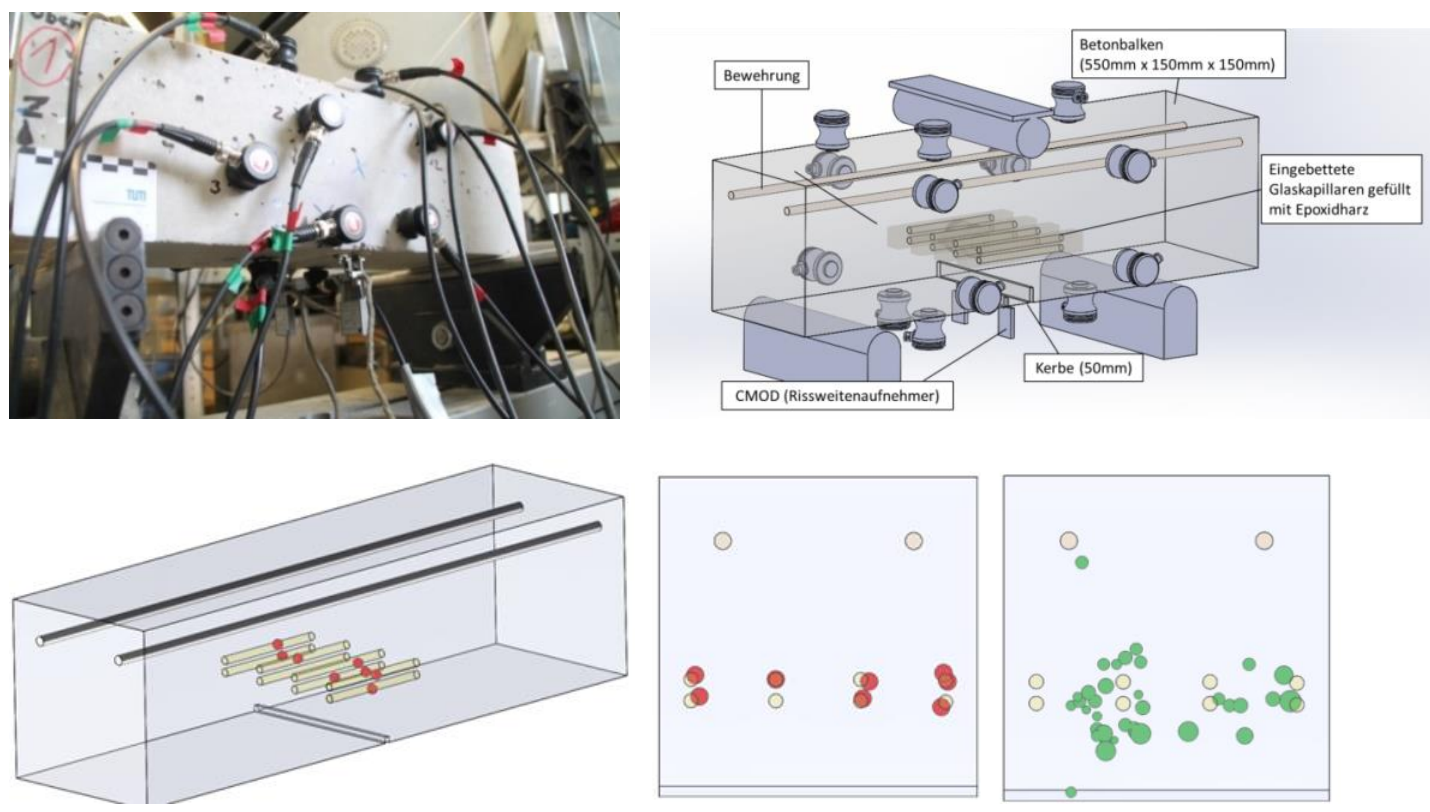

Figure 3. Monitoring of acoustic emission during the three-point bending test of a 
reinforced concrete specimen with encapsulated adhesive self-healing components (top). Prove and localization of glass-capsule opening (acoustic emission source marked by red dots, bottom), as well as detaching of cured epoxy resin healing agent from crack flanks caused by the increase of the mechanical load (green dots, bottom).

Acoustic emission analysis is also applied to monitor the activation of self-healing agents and to verify the force-locking connection of crack flanks by self-healing adhesive polymers. The test specimen, depicted in Figure 3, comprises eight glass capillaries embedded in the concrete specimen at the expected fracture zone. The initiated cracks propagate and hit these capillaries during the three-point bending test. A further load increase causes the breakage of the glass capillaries. The epoxy resin is released, infiltrates the concrete crack and cures for healing of the structure. Real-time analysis of the emitted acoustic waves by evaluation of the onset of $\mathrm{p}$-wave detection allows for localization of the acoustic emission and therefore monitoring of the described healing process. Even for further load increase after healing acoustic emissions are recorded, which can be assigned to the detaching of cured polymer from the crack flanks (Fig. 3, bottom).

Parameter studies for different load-scenarios (overall load, load steps, deflection, fracture width) and different healing-agents can be conducted by acoustic emission analysis for the development of self-healing concrete structures. The localization accuracy is, however, limited due to the assumption of a linear travel path of the pwave front from the emitting source towards the sensor. For damaged concrete specimen multiple scattering of the $\mathrm{p}$-wave is caused, resulting in a deviation from the linear travel path. Onset evaluation for AEA becomes inconclusive and must be discussed regarding the achievable localization accuracy.

\section{Ultrasound Time of Flight Analysis}

Acoustic waves initiated and received by ultrasound transducers in transmission mode through the inspection area are used to detect small cracks in cementitious materials (Hilloulin et al., 2014) and to analyse crack growth (Van Hauwaert et al., 1998). A continuous inspection during a mechanical or thermal load scenario is applied for monitoring the status of damage. A typical active test setup for the ultrasound travel time analysis (transmission mode), applicable also during the mechanical load scenario of a three point-bending test, is shown in Figure 4.

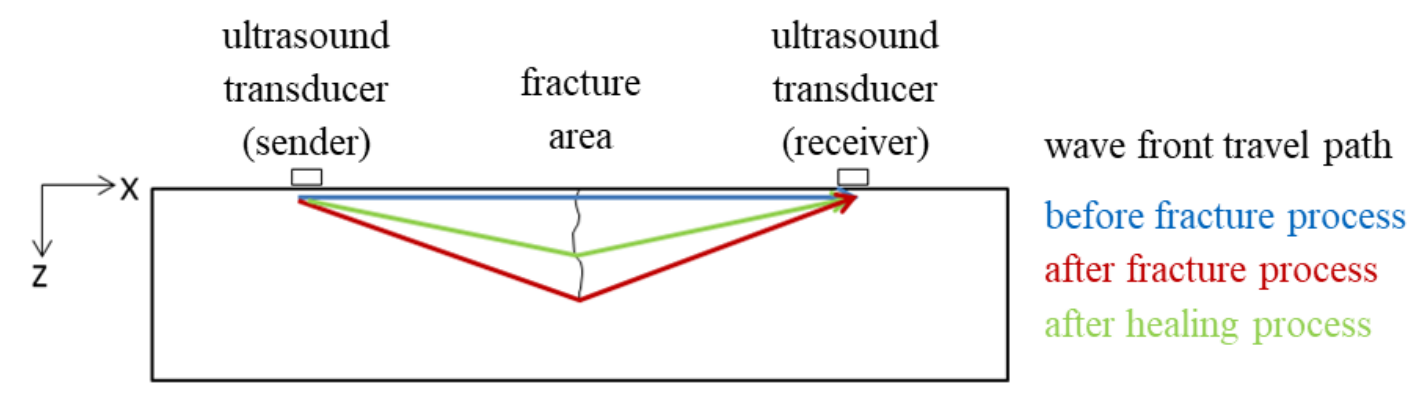

Figure 4. Inspection of crack formation and self-healing by means of ultrasound travel time investigation. Prove of force-locking connection of crack flanks.

An ultrasound transducer (transmitter) initiates a mechanical pulse, whereas the second ultrasound transducer (receiver) detects the propagated mechanical wave. Both, transmitter and receiver are defined in location and time. Referencing the wave front travel time (path) before the fracture process and discussing any deviation of the first on-set being caused by a vertical crack within the fracture area (Fig. 4) enables the detection of small cracks and characterisation of the vertical dimension of the crack.

For the active inspection using ultrasound signals, the inspection frequency must be carefully chosen. Due to multiple-scattering in heterogeneous cementitious materials the frequency range should be less than $400 \mathrm{kHz}$. Furthermore, the analysis of 
ultrasound travel times (ToF) by the evaluation of the onset of the p-wave, is limited in accuracy, as discussed earlier, due to the assumption of a linear travel path of the wave front from the emitting source to the end of the crack and towards the sensor. However, depending on the concrete specimen production process, heterogeneities causing the inaccuracy may differ depending on the depth of travel z (Fig. 4). For ultrasound ToF analysis this inaccuracy must be discussed regarding the achievable crack detection and sizing accuracy. Furthermore, cracks of different expansions within the three dimensions of the inspection area might be underestimated by taking only the shortest wave path into account for the time of flight analysis.

\section{Ultrasound Signal Correlation Analysis}

Test procedures, as the acoustic emission and ultrasound travel time analysis, are established to monitor structural components while damage occurs as well as before and after the damage process. The investigation of heterogeneous materials is, however, limited in detectable microscopic defect sizes and number of occurring microscopic defects by the determination of changes in travel time. For concrete specimens, the wavelength of the propagating waves can be chosen in the same order of magnitude as the size of heterogeneities. Therefore, multiple-scattering effects occur resulting in a diffuse wave field rather than a direct wave path. Beside the described investigation of the travel time by picking the onset of the detected wave mode, a comparison of a $200 \mu$ s time window of the recorded wave propagation is investigated to locate weak perturbations in a multiple scattering environment.

For ultrasound signal correlation analysis, a mechanical load scenario, affecting the structure of concrete railway sleepers, is described in the following. The fracture area and sensor placement for ultrasonic transmission measurements is shown in Figure 5.
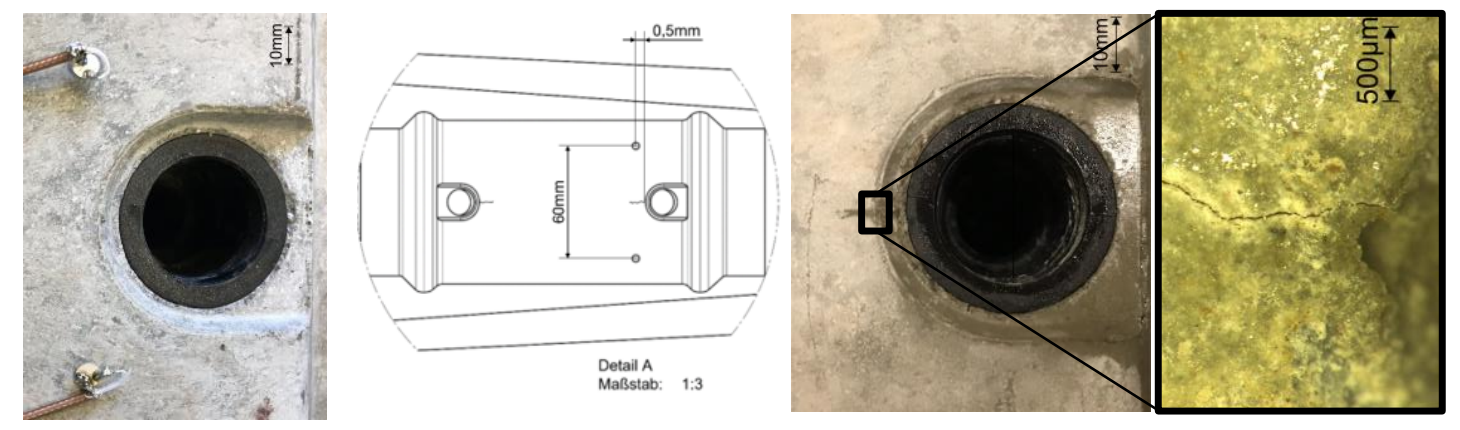

Figure 5 Sensor placement for the inspection of the concrete component by ultrasonic transmission measurement before coupling (left). Dimensioning of the inspection area (middle). Case of damage caused by the mechanical load scenario (right).

Measurements in terms of wave propagation in concrete, therefore ultrasound and acoustic emission analysis tests in general, are identified to be very sensitive to temperature variations. Due to the strong dependency of different wave regimes on the temperature (Zhang et al., 2016), the specimen for the experimental procedure of ultrasound correlation analysis is tempered at $20{ }^{\circ} \mathrm{C}$ for 72 hours before testing. A single load state is characterized by a specific, manually applied torque (Fig. 6, top). The torque increment from one to another load step is set constant. Ultrasonic transmission measurements are performed at discrete load states with a defined torque (Fig. 6, bottom). The pulse excitation is designed as a burst with a duration of $2 \mu s$ and a maximum amplitude of $350 \mathrm{~V}$. The short burst duration is chosen to create a broadband excitation, while the amplitude was determined iteratively taking a SNR of at least $10 d B$ as a basis within the response signal (Fig. 6, middle).

The ultrasonic signals recorded at different load and respectively damage states are correlated and evaluated by means of the Pearson correlation coefficient. The experiment is divided into two periods, the incremental load application and the long- 
term observation of the relaxation process (Fig. 6, middle). Every measurement series is considered compatible to clustering as the variations within one measurement series are small compared to the change between two measurement series. In this measurement, the correlation coefficient for the first series is approximately equal to 1 , while the mean value of every series decreases continuously with increasing load.
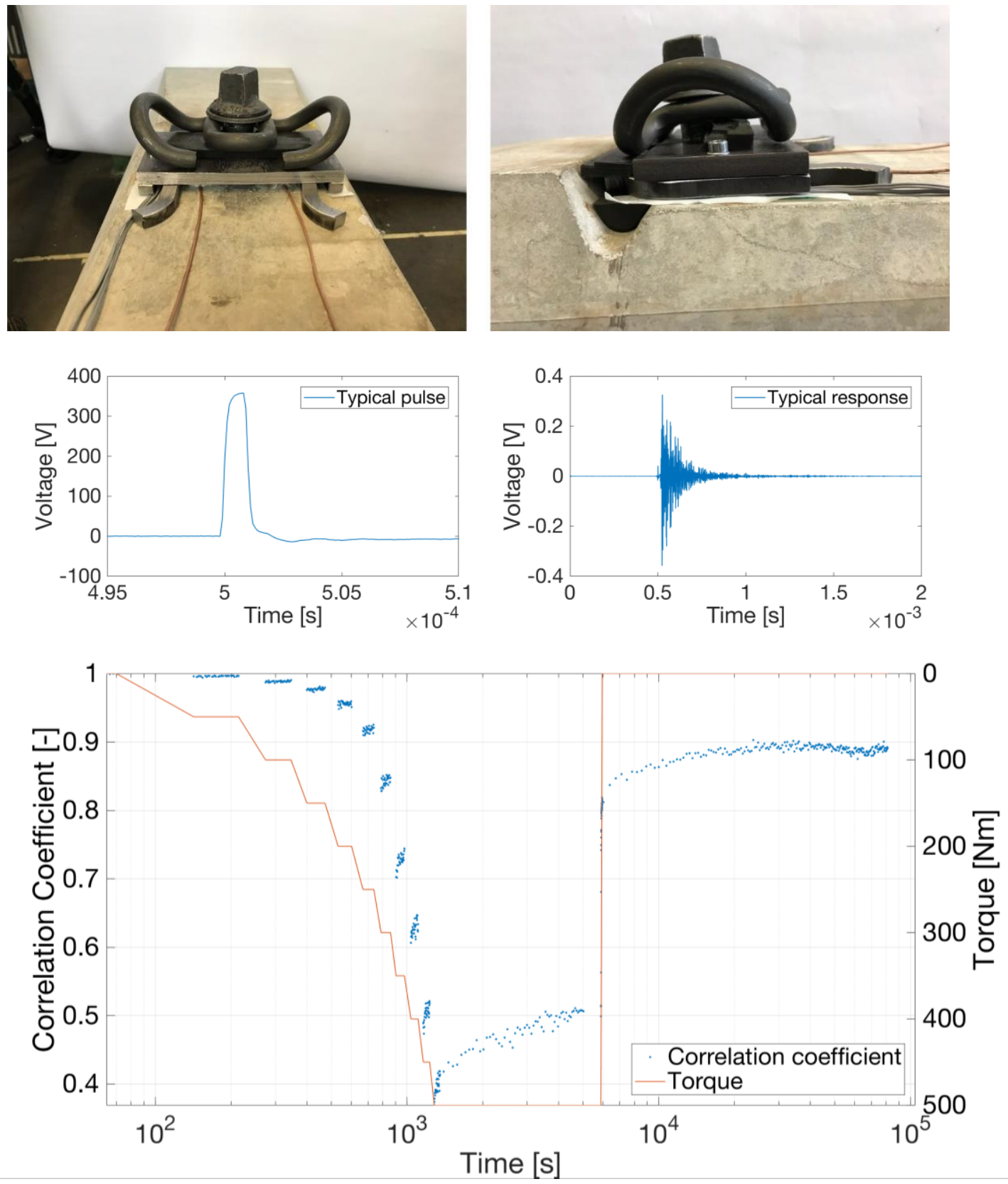

Figure 6. Mechanical load scenario, defined by a specific, manually applied torque (top). Signal and response characteristics of the reference ultrasound transmission measurement (middle). Result of the correlation analysis. Correlation coefficient and applied torque in reference to the timeline of the load experiment (bottom). Correlation coefficient corresponding to a gradual load application up to $500 \mathrm{Nm}$, unloading (at $\sim 6^{*} 103 \mathrm{~s}$ ) and long-term relaxation process over logarithmic time.

Also, the difference between two correlation coefficient means at discrete load states tends to increase for greater torques. The mean difference between $0 \mathrm{Nm}$ and $50 \mathrm{Nm}$ torque is 0.01 , whereas the difference between $300 \mathrm{Nm}$ and $350 \mathrm{Nm}$ is 0.11 . The minimum mean for $500 \mathrm{Nm}$ is reached at 0.41 . During the unloading process with a duration of $60 \mathrm{~s}$, an abrupt increase of the correlation coefficient from 0.50 to 0.82 is observed indicating reversible structural changes. Furthermore, an ongoing regression and an asymptotic approximation to the value 0.89 is observed within the time span of 24 hours. As the damage level of the specimen mostly depends on the applied torque, 
damage level and correlation coefficient are evaluated to have a functional interrelationship. This relationship is very complex since further parameters have an impact on the signal, e.g. former prestressing, temperature or moisture (Niederleithinger et al. 2018).

In addition to the evaluation of structural damage caused by the mechanical load scenario, the effect of an applied hydrophobic healing polymer on pre-existing as well as on enforced microcracks is examined. A healing agent is applied on the specimen (Fig. 7, left and right) after the load induced damage and a load scenario with quasicontinuous ultrasonic transmission measurement is performed (Fig. 8). The polymer's intrusion into the cracks is analysed by ultrasonic measurement and by a light optical microscope (Fig. 7, middle). The bonding condition between the intruded polymer and the concrete sleeper is assessed on a microscale level.
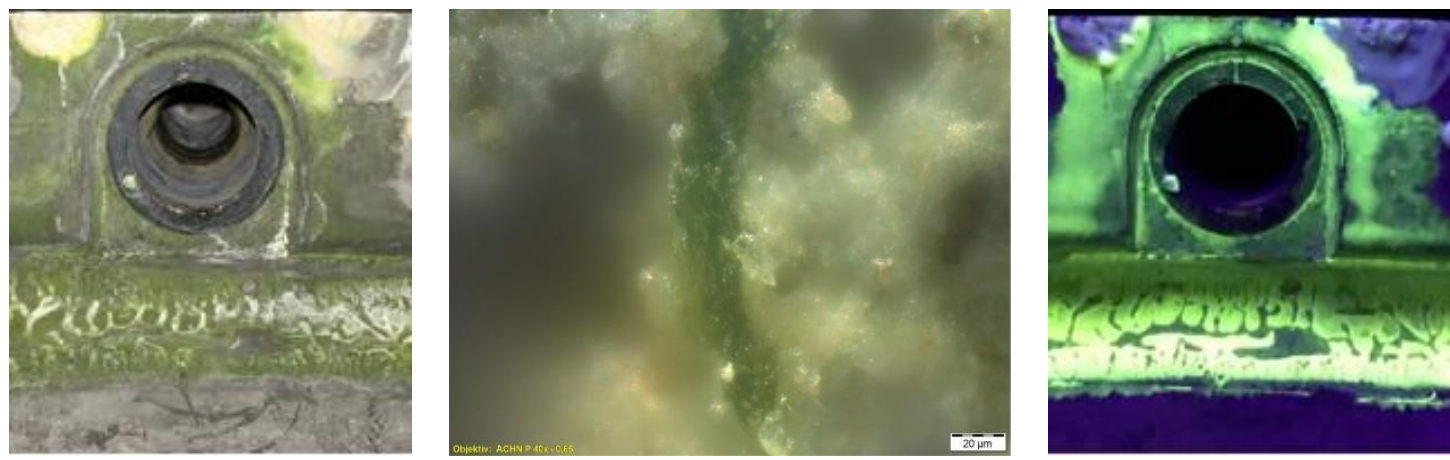

Figure 7. Applied self-healing agent. Polymer propagation around the dowel (left) and into the microcrack (middle). Visualization of the propagation zone using UV radiation (right, fluorescent particles were added to the self-healing polymer before application).

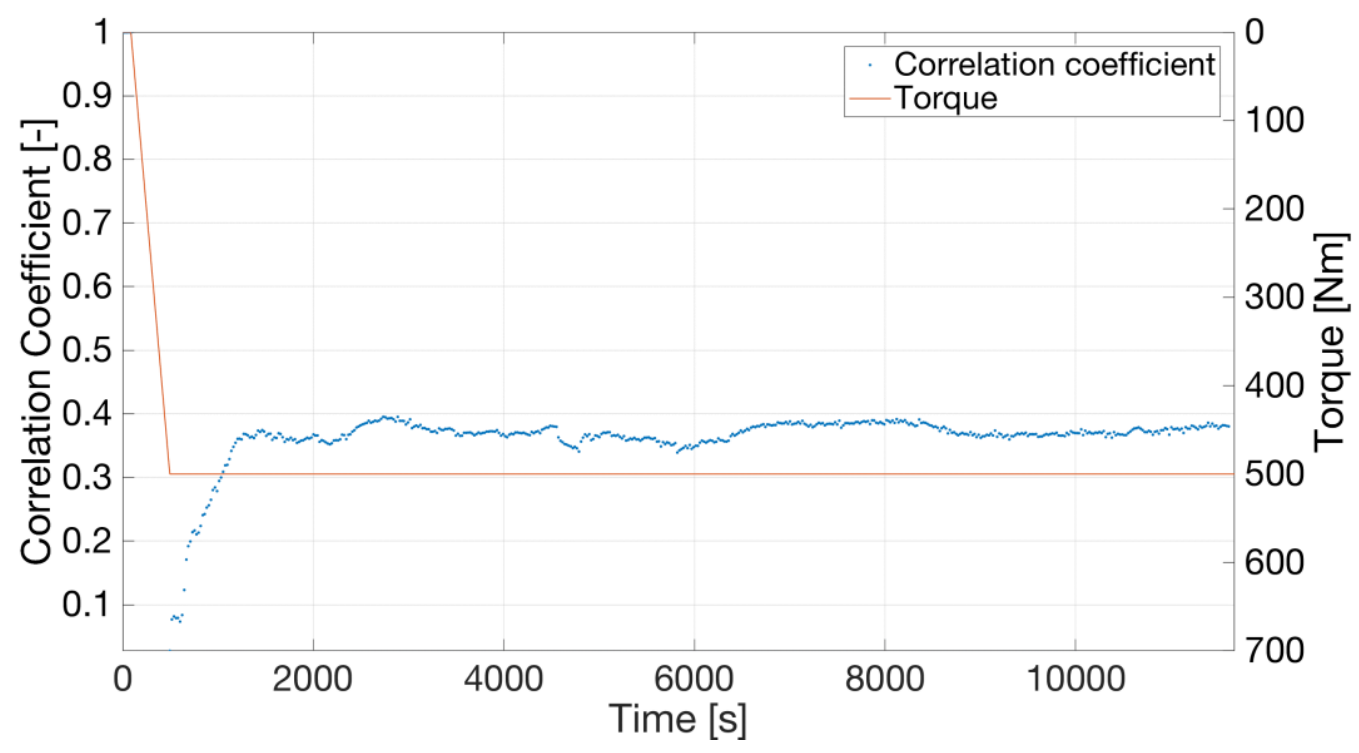

Figure 8. Correlation coefficient corresponding to the application of the self-healing agent and the application of a torque of $500 \mathrm{Nm}$ for $3.2 \mathrm{~h}$.

Five signals are recorded as a reference at unloaded state. After a torque application of $500 \mathrm{Nm}$, the specimen is coated with the healing agent around the dowel opening and onto the initial microcracks, which originate radially from the dowel in the specimen's longitudinal direction.

The application of the healing polymer immediately caused a significant increase and large variation of the correlation coefficient. The effect is assessed to be composed of two mechanisms, first, additional elastic wave propagation within the applied polymer. And second, wave propagation changes during the curing process of the healing agent. 
These effects may not be explicitly separated and must be considered to have interdependency in terms of interpreting the healing agent effect.

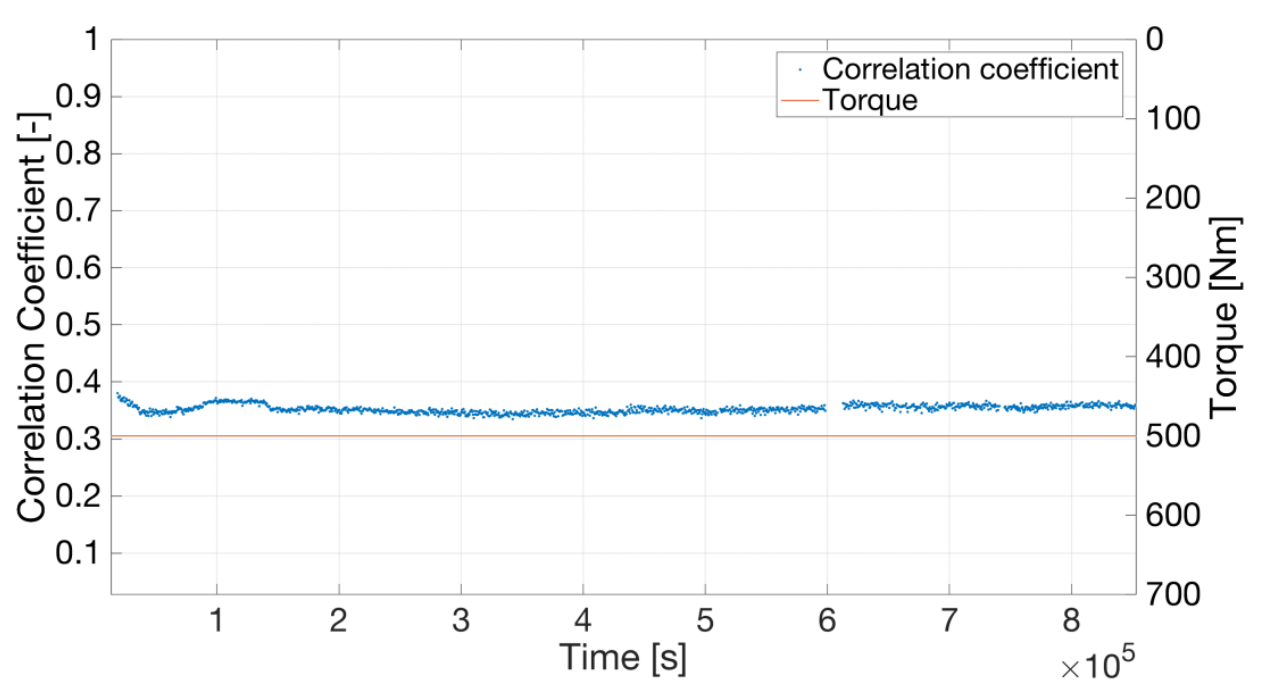

Figure 9. Correlation coefficient corresponding to long term observation for 9.5 days.

After ten days, the healing agent was assessed to be cured. The correlation coefficient of the healed specimen showed a different behaviour compared to a sound specimen.

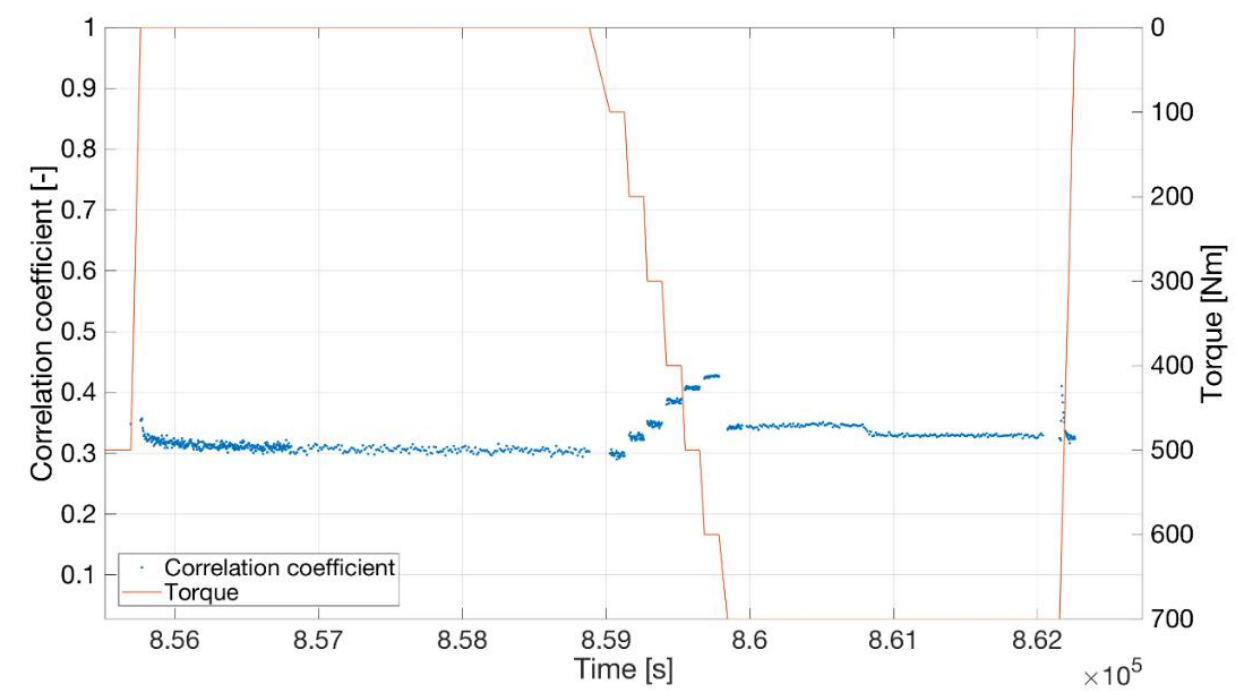

Figure 10. Correlation coefficient corresponding to unloading, reloading up to $700 \mathrm{Nm}$ and re-unloading.

The third period covers the correlation coefficient development immediately after unloading ( 9.5 days). Compared to the experiments that were conducted beforehand, only a relatively small drop is observed. The specimen is reloaded up to $700 \mathrm{Nm}$ and held at that level of mechanical load for half an hour. During that period, another drop of the signal is observed, though, the loading state was not changed externally. The observed effects may be interpreted by the characteristics of the intruded polymer (compaction due to tension, tearing effects at load levels above $500 \mathrm{Nm}$ ) but must be verified by additional experiments.

\section{CONCLUSION AND OUTLOOK}

The detailed study of self-healing processes in building materials aims at the development of novel sustainable and durable construction materials. The experimental setup of acoustic emission analysis and ultrasonic transmission testing is 
proven to reproducibly inspect and monitor construction structures during mechanical load scenarios and self-healing processes. The evaluation of the onset of the detected wave mode (time of flight analysis) is, however, limited by the assumption of a linear travel path of the p-wave front from the emitting source towards the sensor. In comparison, the evaluation of the correlation of specified time windows of the recorded wave propagation signals is presented. The applied linear correlation analysis is a simple-to-implement form of coda wave interferometry. Different load and therefore states of damage, including the initial micro crack formation, as well as the self-healing behaviour are characterized by a change of the calculated linear correlation coefficient. However, due to its mathematical character, the correlation coefficient is only suitable to measure the linear correlation between two signal forms. A multi-parameter study might reveal more complex co-dependencies and is necessary to take the next step towards predictive maintenance of construction structures. 


\section{REFERENCES}

De Rooij, M., Van Tittelboom, K., De Belie, N., Schlangen, E. (2013). "Self-Healing Phenomena in Cement-Based Materials" Springer Science+Business Media, Dordrecht.

Feiteira, J., Gruyaert, E., De Belie, N. (2015). "Self-healing of moving cracks in concrete by means of encapsulated polymner precursors" Construction and Building Materials, 102, 671-678.

Flohr, K., Malm, F., Grosse, C. U. (2017). "Schallemissionsanalyse zur Untersuchung der Effizienz von Selbstheilungsmechanismen in Beton" 20. Kolloquium Schallemission, Garmisch-Patenkirchen.

Hilloulin, B., Zhang, Y., Abraham, O., Grondin, F., Loukili, A., Durand, O., Tournat, V. (2014). "Small crack detection in cementitious materials using nonlinear coda wave modulation" NDT\&E International, 68, 98-104.

Hilloulin, B., Hilloulin, D., Grondin, F., Loukili, A., De Belie, N. (2015). "Mechanical regains due to self-healing in cementitious materials: Experimental measurements and micro-mechanical model" Cement and Concrete Research, 80, 21-32.

Niederleithinger, E., Wang, X., Herbrand, M. Müller, M. (2018). "Processing Ultrasonic Data by Coda Wave Interferometry to Monitor Load Tests of Concrete Beams" Sensors, 19, 147.

Planès, T., Larose, E. (2013a). "A review of ultrasonic Coda Wave Interferometry in Concrete" Cement and Concrete Research, 53, 248-255.

Planès, T., Larose, E., Rossetto, V., Margerin, L. (2013b). "LOCADIFF: Locating a weak change with diffuse ultrasound" The 39th Annual Review of Progress in Quantitative Nondestructive Evaluation, AIP Conf. Proc. 1511, 405-411.

Snoeck, D., Malm, F., Cnudde, V., Grosse, C. U., Van Tittelboom, K. (2018). "Validation of Self-Healing Properties of Construction Materials through Nondestructive and Minimal Invasive Testing" Adv. Mater. Interfaces, 5, 1800179, 28 p.

Van Hauwaert, A., Thimus, J.-F., Delannay, F. (1998). "Use of ultrasonics to follow crack growth" Ultrasonics, 36, 209-217.

Zhang, M., Han, N., Xing, F., Schlangenm H. E. J. G. (2013) "Design of microcapsule system used for self-healing cementitious material" Proceedings of the 4th International Conference on Self-Healing Materials, Ghent, Belgium.

Zhang, Y., Tournat, V., Abraham, O., Durand, O., Letourneur, St., Le Duff, A., Lascoup, B. (2016) "Nonlinear coda wave interferometry for the global evaluation of damage levels in complex solids" LUNAM Université, France. 